\title{
Ebola virus disease in West Africa - an unprecedented outbreak
}

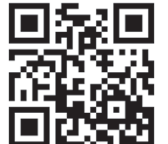

Ebola virus disease (EVD) is one of the most feared diseases known to mankind. This is because of the high mortality rate (up to $90 \%$ ) associated with the disease, and also its propensity for person-to-person spread through close contact with infected tissues and body fluids of affected persons, particularly in the unprotected home-care setting and during preparation of bodies for burial, while spread is amplified in hospitals with poor infection control practices. The disease has nevertheless remained rare since its initial description in 1976, with no more than 2000 cases diagnosed before 2014. ${ }^{[1]}$ This is why the current outbreak of EVD in West Africa is of particular concern. Not only has the outbreak been raging for more than 6 months - to date, more than 800 cases of EVD have been recorded in the three affected countries of Guinea, Liberia and Sierra Leone ${ }^{[2]}$ - but it accounts for more than a third of the cases of EVD ever reported. Indications are that at the time of writing the outbreak is far from over. It is also noteworthy that this is the first recorded outbreak of EVD in this region of Africa. Historically EVD has been recorded in several central African countries, including the Democratic Republic of Congo (DRC), Sudan, Gabon, Uganda and Congo, with cases imported to South Africa (SA), The Netherlands, Italy and the USA..$^{[1-3]}$

The current outbreak was officially reported by the World Health Organization (WHO) on 23 March 2014. Contact tracing has led to an index case involving a 2-year-old child who died on 6 December 2013 in the Guéckédou Prefecture of Guinea. ${ }^{[4]}$ To date, a total of 408 cases from Guinea, 305 from Sierra Leone and 131 from Liberia have been recorded ${ }^{[2]}$ The total case tally is probably underestimated owing to inaccessibility of some of villages in the affected areas and reluctance to co-operate on the part of the local communities. The outbreak appeared to have started to wane in April, with certain affected areas not reporting any cases for weeks, but resurged in May, setting off a second wave of cases reported in Sierra Leone and Liberia, and again in Guinea. ${ }^{[2,5,6]}$

The West African outbreak has proved particularly challenging to contain. ${ }^{[6,7]}$ Certainly the extent of its spread across three countries in itself complicates all efforts, as co-ordination must be achieved across three separate healthcare jurisdictions involving the different healthcare authorities and role players in each of the countries. The affected regions generally represent resource-poor settings, with healthcare systems struggling to meet the everyday healthcare needs of the communities they serve. A haemorrhagic fever outbreak requires a comprehensive and intensive public health response that would put even an ideal healthcare system to the test. Substantial external support is always required during these events to ensure appropriate clinical management of patients, sufficient infection control procedures in difficult healthcare environments, intensive contact tracing to identify the chains of transmission of the virus (key to eventually containing the disease from further spread), appropriate risk communication to the affected communities, and comprehensive logistical support to tie everything together. Specialised and rapid laboratory responses are required to confirm or exclude diagnosis in suspected cases. Laboratory testing in this setting must also provide a differential diagnosis for Lassa fever, a haemorrhagic fever endemic to and commonly reported from the affected areas, among other likely causes of disease that may present with similar clinical features. Community perceptions, fears and reluctance to co-operate can be especially problematic, and communication barriers must be bridged by well-designed and understandable health messages. The prolonged nature of the outbreak is also imposing a burden on the support teams that are expected to work long hours away from home. A summit was co-ordinated by the WHO in Ghana in July 2014 to address these very issues, strategise resources for the months ahead, and put an action plan on the table. ${ }^{[6]}$

EVD is caused by the Ebola virus, a filovirus that is thought to be harboured by specific arboreal bat species in the affected regions. ${ }^{[1]}$ The current theory regarding the ecology of the virus holds that the virus is harboured by these bat species (bat-to-bat transmission, pathology and immunity features in bats are still to be definitively described) with incidental spillover (mechanism of transmission still to be proven) of the virus to other animal species (various species of antelope and primate have been found to be infected with Ebola virus). The virus may then spill over to the human population through contact between infected animals and humans, or direct contact with infected bats. The mode of transmission to the human population remains to be elucidated. Once the virus has entered the human population, outbreaks of EVD are often characterised by transmission of the virus in close family clusters and in the nosocomial setting. ${ }^{[1]}$ Five strains of Ebola virus, which are genetically and antigenically distinct, have been identified over the years. These include the Tai Forest, Reston, Sudan, Zaire and Bundibudyo viruses (Bundibudyo virus was identified for the first time during an outbreak in Uganda in 2008). The latter three strains have been associated with sizeable outbreaks in sub-Saharan Africa, with mortality rates of $30-90 \%$. These viruses differ in geographical spread, and the Zaire Ebolavirus has been associated with the most fatal outbreaks to date ${ }^{[1]}$ The mortality rate of the current outbreak fluctuates with the identification of new cases, but is reported to be in the range of $60-70 \%{ }^{[2,6]}$ The current outbreak is caused by a variant of Zaire Ebolavirus with $97 \%$ sequence identity to strains isolated from the DRC and Gabon, suggesting a parallel evolution of this virus in the affected area as opposed to introduction from these endemic areas ${ }^{[4]}$ Common clinical features of patients during this outbreak have included fever and severe diarrhoea with vomiting; however, bleeding manifestations have not been a regular finding. ${ }^{[4]}$ Generally, early signs and symptoms of EVD are very nonspecific and need to be differentiated from other causes of acute febrile illness that occur in the area, notably malaria, typhoid and bacterial dysentery. The typical signs and symptoms of EVD include fever, chills, malaise, myalgia and often a maculopapular rash. The disease progresses rapidly to multisystem involvement that may include systemic, gastrointestinal, respiratory and vascular features. ${ }^{[1]}$ Patients may present with an apparent acute abdomen, and the surgical interventions that may follow represent a further risk for infection on the part of healthcare workers. Haemorrhagic manifestations, if present, may include petechiae, ecchymoses, bleeding from venepuncture sites and mucosal haemorrhages. Clinical management of cases involves employing strict isolation procedures and providing symptomatic and supportive treatment as required. No specific antiviral treatments or pre- or post-exposure vaccines have been approved or registered for use against EVD. Various drugs and vaccine candidates are reportedly at various stages of evaluation. ${ }^{[1]}$ Interestingly, ribavirin which is broadly effective against many haemorrhagic fever viruses, appears to have no effect on Ebola virus. ${ }^{[8]}$

To date, no cases of EVD related to this outbreak have been reported in or confirmed to have been exported to SA. In 1996 EVD 


\section{EDITORIAL}

was confirmed in a nurse working in a Johannesburg hospital, who was probably exposed during a procedure performed on a medical practitioner who was being investigated for an unknown febrile illness with jaundice. He presented to the Johannesburg facility as a self-referral from his work place in Libreville, Gabon. The patient was probably infected while caring for patients during an EVD outbreak in Gabon at that time. Tragically, the nurse died, and the diagnosis of EVD was confirmed retrospectively on the doctor, who survived. ${ }^{[3]}$ The risk of EVD in SA travellers to the regions affected by the current outbreak would be considered low, given that casual contact with infected persons does not pose a risk. However, although malaria is much more likely, a high index of suspicion needs to be in place for EVD, or other haemorrhagic fevers such as Lassa fever, in any patient presenting with an unexplained fever who has been travelling in the affected regions. Healthy persons returning from these areas do not require quarantine. The National Institute for Communicable Diseases has the capacity for rapid, reliable and specialised differential diagnoses of viral haemorrhagic fevers, including EVD, which is integral to the response to any suspected case(s) that may present to SA healthcare facilities.

\section{Jacqueline Weyer}

Centre for Emerging and Zoonotic Diseases, National Institute for Communicable Diseases, National Health Laboratory Service, Sandringham, Johannesburg, South Africa

\section{Lucille H Blumberg}

Division for Surveillance and Outbreak Response, National Institute for Communicable Diseases, National Health Laboratory Service, Sandringham, Johannesburg, South Africa

\section{Janusz T Paweska}

Centre for Emerging and Zoonotic Diseases, National Institute for Communicable Diseases, National Health Laboratory Service, Sandringham, Johannsburg, South Africa

\section{Corresponding author: L H Blumberg (lucilleb@nicd.ac.za)}

\footnotetext{
1. Feldmann H, Geisbert TW. Ebola haemorrhagic fever. Lancet 2011;337:849-862. [http://dx.doi org/10.1016/S0140-6736(10)60667-8]

2. Centers for Disease Control and Prevention. Outbreak of Ebola in Guinea, Liberia and Sierra Leone. http://www.cdc.gov/vhf/ebola/outbreaks/guinea/ (accessed 9 July 2014).

3. Richards GA, Murphy S, Jobson R, et al. Unexpected Ebola virus in a tertiary setting: Clinical and epidemiological aspects. Crit Care Med 2000;28(1):240-244. [http://dx.doi.org/10.1097/00003246200001000-00041]

4. Baize S, Pannetier D, Oestereich L, et al. Emergence of Zaire Ebola Virus disease in Guinea - preliminary report. N Engl J Med 2014. Epub: 16 April 2014. [http://dx.doi.org/10.1056/NEJMoa 1404505]

5. Dixon MG, Schafer IJ. Ebola viral disease outbreak - West Africa 2014. MMWR Morb Mortal Wkly Rep 2014;63(25):548-551.

6. World Health Organization. Ebola virus disease, West Africa updates. http://www.afro.who.int/en/ clusters-a-programmes/dpc/epidemic-a-pandemic-alert-and-response/outbreak-news/4217-ebolvirus-disease-west-africa-7-july-2014.html (accessed 9 July 2014).

7. Waddington C. Ebola outbreak in Guinea: A different type of regional stability threat. Africa Conflict Monthly Monitor 2014;May:47-50.

8. Ignatyev G, Steinkasserer A, Streltsova M, et al. Experimental study of possibility of treatment of some 8. Ignatyev G, Steinkasserer A, Streltsova M, et al. Experimental study of possibility of treatment of some
haemorrhagic fevers. J Biotechnol 2000;83:67-76. [http://dx.doi.org/10.1016/S0168-1656(00)00300-X]
}

S Afr Med J 2014;104(8):555-556. DOI:10.7196/SAMJ.8672 